\title{
Estudio preliminar de las características del intento de suicidio en la provincia de Granada
}

\author{
Preliminary study of the characteristics of attempted \\ suicide in the province of Granada
}

\author{
Y. Mejías ${ }^{1}$, M.P. García Caro ${ }^{2}$, J. Schmidt ${ }^{2}$, A. Quero ${ }^{3}$, B. Gorlat ${ }^{1}$
}

\section{RESUMEN}

Fundamento. Los suicidios en España representan un número de muertes mayor que otras muertes traumáticas. Los comportamientos suicidas no mortales se producen en una proporción mayor que los que tienen fin mortal. Las causas de esta conducta son múltiples y es importante conocerlas para poder prevenirlas. El objetivo del presente trabajo es describir las características del comportamiento suicida no mortal en la provincia de Granada.

Método. Estudio descriptivo retrospectivo de las características del comportamiento suicida no mortal en Granada durante los años 2008 y 2009, según la base de datos del Servicio Provincial 061 de Granada. La muestra ha sido todas las demandas realizadas a este servicio en las que en el motivo literal de la alerta figuraron los términos suicidio, autolesión o amenaza suicida. El análisis estadístico se ha realizado con el programa SPSS 15.0. Se analizó la variable conducta suicida no mortal respecto de las variables independientes: sexo, edad, mes, día de la semana, franja horaria, distrito sanitario, resolución de la demanda (prioridad asignada) y reintentos.

Resultados. Se analizaron en total 535 demandas tipificadas como lesión autoinflingida intencionalmente por medios no especificados. Se han encontrado diferencias estadísticamente significativas al comparar los datos de los dos años respecto del mes elegido, y en la relación entre rango de edad y franja horaria de los intentos de suicidio.

Conclusiones. La caracterización de los intentos de suicidio en nuestra provincia ofrece información relevante, a pesar de sus limitaciones, y ayuda a determinar alguno de los parámetros en los que habrá que sustentar un futuro programa de intervención psicosocial para la prevención de estas conductas ajustado a los perfiles específicos de nuestra población.

Palabras clave. Estudio retrospectivo. Intento de suicidio. Factores de riesgo.

\begin{abstract}
Background. Suicides account for a number of deaths that is higher than other traumatic deaths in Spain. Non-fatal suicidal behaviour occurs in a greater proportion than such behaviour with a deadly outcome. There are many causes for this behaviour and it is important to become familiar with them if it is to be prevented. The aim of this article is to describe the characteristics of non-mortal suicidal behaviour in the province of Granada.
\end{abstract}

Method. A retrospective descriptive study of the characteristics of non-mortal suicidal behaviour in Granada during the years 2008 and 2009, according to the database of the Provincial 061 Service in Granada. The sample includes the demands made to this service in which the literal reason for the alert included the terms: suicide, self-harm or the threat of suicide. The statistical analysis was done with the SPSS 15.0 program. We analyzed the variable non-mortal suicidal behaviour with respect to the independent variables: sex, age, month, day of the week, time slot, health district, resolution of the demand (assigned priority) and further attempts.

Results. In total we analyzed 535 demands typified as intentional self-injury by unspecified means. Statistically significant differences were found when comparing the data for the two years with respect to the month chosen, and in the relationship between age range and time slot of the suicide attempts.

Conclusions. Despite its limitations, the characterization of suicidal behaviour in our province offers essential information, and could be useful in designing and developing a program of psychosocial intervention for the prevention of suicidal behaviour adjusted to the specific profiles of our population.

Key words. Retrospective study. Attempted suicide. Risk factors.
1. Hospital Universitario Virgen de las Nieves. Granada.

2. Facultad de Ciencias de la Salud de la Universidad de Granada.

3. Escuela Universitaria de Enfermería Virgen de las Nieves. Granada.

Recepción: 13 de mayo de 2011

Aceptación provisional: 11 de julio de 2011

Aceptado definitiva: 24 de agosto de 2011

\section{Correspondencia:}

Yolanda Mejías Martín

Hospital Universitario Virgen de las Nieves 18008 Granada

E-mail. yolandamejiasmartin@yahoo.es 


\section{INTRODUCCIÓN}

Las políticas sanitarias y sociales actuales dirigen sus esfuerzos tanto a prevenir muertes accidentales como a disminuir el efecto de los accidentes en la calidad de vida de las personas sanas. Algunos ejemplos son la Ley sobre Tráfico, Circulación de Vehículos a Motor y Seguridad Vial; la Ley de Prevención de Riesgos Laborales; la Ley frente al tabaquismo y reguladora de la venta, el suministro, el consumo y la publicidad de los productos del tabaco; la Ley contra la venta y publicidad de bebidas alcohólicas a menores, etc. Son medidas que desarrollan planes de prevención que ayudan a proteger de los riesgos y ofrecen información sobre los efectos de un mal uso de sustancias y elementos habituales en nuestro devenir diario.

Uno de los principales problemas de salud pública de mayor impacto, tanto por los costes económicos como por los sociales, sanitarios y personales, es el suicidio.

Este acto si lo comparamos, en número de muertes producidas, con otros sucesos traumáticos que tienen el mismo fin mortal, observamos que en España, en el año 2008, hubo 1.065 accidentes laborales mortales (Ministerio de Trabajo e Inmigración), 2.181 víctimas mortales en accidentes de carretera (Dirección General de Tráfico), y 3.457 suicidios (Instituto Nacional de Estadística).

El suicidio es una de las 20 principales causas de mortalidad a nivel mundial para todas las edades ${ }^{1}$. En el periodo de edad comprendido entre los 15-44 años, se encuentra entre la cuarta causa de muerte y la sexta como causa de mala salud y discapacidad $^{2}$, reafirmando el problema de salud pública que puede llegar a suponer, especialmente entre los jóvenes.

La Organización Mundial de la Salud y la Organización Panamericana de la Salud estimaron que en el año 2000 se produjo una muerte por suicidio cada 40 segundos y un intento de suicidio cada tres ${ }^{3}$. El riesgo de presentar conductas precursoras del suicidio aumenta a medida que disminuye la edad, los más jóvenes son los que presentan más riesgo de elaborar ideas o planes de suicidio y realizar tentativas de suicidio $^{4,5}$. El estudio de Gabilondo ${ }^{4}$, en la población general española, demuestra que las personas que han tenido ideas de muerte y han elaborado un plan de suicidio tenían una probabilidad superior a los que no lo habían tenido. En este estudio se señala que la probabilidad de progresión de la idea al plan o al intento es mayor en el primer año de aparición de la idea. El riesgo de ideación o elaboración de un plan suicida es mayor en las mujeres que en los hombres aunque no presentan mayor riesgo de intentarlo. Igualmente se confirma la relación en un $90 \%$ de los casos entre suicidio y trastorno mental.

Sólo en el servicio de Urgencias del centro Médico-Quirúrgico del Hospital Universitario Virgen de las Nieves de Granada, que da cobertura al área norte de la provincia, con una población de 439.035 personas, se atendieron en el año 2008 y en el 2009, por autolesión deliberada con o sin resultado mortal, a 276 y 184 personas respectivamente.

El suicidio es un fenómeno complejo y de etiología multifactorial, entre las causas destacan tener una enfermedad mental ${ }^{6}$, padecer enfermedades crónicas o discapacidad física ${ }^{7}$ tener antecedentes de un intento anterior ${ }^{8,9}$, acontecimientos de la vida y factores sociales y ambientales como la facilidad de disponer de los medios para realizar el suicidio ${ }^{10}$. Por lo tanto, un programa para la prevención del suicidio debe contemplar la suma de diversas intervenciones. Por ejemplo, en Dinamarca, se ha reducido hasta un $60 \%$ las tasas de suicidio con un programa de prevención en el que combinan medidas como reducción del acceso a los medios para cometer suicidio, un mejor tratamiento psiquiátrico y físico después de un intento de suicidio, una mayor estabilidad social y cultural, un mayor esfuerzo en prevención y un mejor acceso a los servicios de asesoramiento telefónico y los servicios de urgencias $^{11}$.

Un factor predictivo del suicidio es tener antecedentes de un intento anterior $^{4,5,8,9}$, la magnitud de los reintentos justifica que una vez identificadas las personas que han realizado una conducta suicida no mortal se realice una intervención para 
evitar otro intento de suicidio que puede tener, en otra ocasión, un final mortal.

Por tanto se justifica la importancia de conocer las características específicas de los grupos de riesgo para desarrollar un programa de prevención y de esta forma investigar y evidenciar qué tipo de intervenciones pueden ayudar a prevenir los suicidios ${ }^{12}$.

El objetivo de este estudio es conocer y describir las características del comportamiento suicida no mortal en la provincia de Granada.

\section{MATERIAL Y MÉTODOS}

Estudio descriptivo retrospectivo de las características del comportamiento suicida no mortal en la provincia de Granada durante los años 2008 y 2009, según la base de datos SIEPES (Servicio Provincial $061 \mathrm{de}$ Granada).

\section{Población y muestra}

La Central de Coordinación de Urgencias y Emergencias del Servicio Provincial 061 coordina todas las urgencias de la provincia y distribuye los recursos según el motivo de la demanda y la prioridad que el médico coordinador les asigne. Estos y otros muchos datos quedan recogidos en la base de datos SIEPES, por lo que constituye la opción más completa para un estudio que incluya por una parte a toda la provincia, y por otra, a todas las demandas, indistintamente de la resolución que pueda tener según la urgencia (atención en domicilio, traslado a urgencias, éxitus...).

De las demandas de asistencia sanitaria realizadas a la Central de Coordinación de Urgencias y Emergencias del Servicio Provincial 061 de Granada, entre el 1 de enero y el 31 de diciembre de 2008 (71.035) y el 1 de enero y el 31 de diciembre de 2009 (78.535), 5.188 llamadas en el año 2008 (7,30\%) y 4.928 en el 2009 (6,27\%), fueron clasificadas como Asistencia Psiquiátrica, Traumatismos e Intoxicaciones y Alergias.

La muestra seleccionada fueron todas las llamadas que la operadora clasificaba como X84 porque en el motivo de demanda aparecía literalmente el término suicidio.
Además se ha depurado la base de datos eliminando las llamadas con el mismo código que se producen en el mismo día y hora (repeticiones).

\section{Análisis de los datos}

El análisis estadístico se ha realizado con el programa SPSS 15.0.

Se analizó la variable conducta suicida no mortal (variable dependiente) respecto de las variables independientes: sexo, edad, mes, día de la semana, franja horaria, distrito sanitario, resolución de la demanda (prioridad asignada) y reintentos.

Se realizó un análisis descriptivo de las diferentes variables, frecuencias y porcentajes (variables cualitativas); medias y otras medidas de dispersión (variables cuantitativas).

Para determinar la relación entre la variable independiente y dependiente, análisis bivariado, se aplicaron diferentes estadísticos en función de la naturaleza de las variables (test de $\chi 2$, t de Student y test de ANOVA). Para todos los contrastes se consideró un nivel de significación de 0,05.

Se analizaron los datos de cada año por separado y posteriormente se compararon ambos años respecto de las variables independientes. Igualmente se estableció la comparación en función del sexo.

Se establecieron rangos tanto para la edad (10-20; 21-30; 31-40; 41-50; 51-60; 61-70; 71-80 y 81-90) como para las horas (08:00$15: 59 ; 16: 00-23: 59 ; 24: 00-07: 59)$, y se analizaron mediante tablas de contingencia.

Las poblaciones de la provincia de Granada se han agrupado según el mapa sanitario de Atención Primaria. Los distritos que la componen son Granada Sur, Metropolitano de Granada, Granada Nordeste y Distrito Granada.

Para obtener los datos relativos a los reintentos, se revisaron en los dos años estudiados las direcciones postales de todas las llamadas de la muestra una vez identificadas las que se repetían, se observaba si eran coincidentes los datos recogidos de la edad y el sexo. Si todas estas características coincidían se aceptaba como reintento de conducta suicida no mortal. 
Tabla 1. Descripción de la muestra según el total y el año

\begin{tabular}{|c|c|c|c|c|c|c|c|c|}
\hline & & & & 20 & & & & $\begin{array}{c}p \\
2008 / 2009\end{array}$ \\
\hline \multirow{2}{*}{ SEXO } & mujer & 280 & $(52,3)$ & 164 & $(51,6)$ & 116 & $(53,3)$ & \multirow{2}{*}{0,719} \\
\hline & hombre & 255 & $(47,7)$ & 154 & $(48,4)$ & 101 & $(46,7)$ & \\
\hline \multirow{8}{*}{ EDAD } & \begin{tabular}{|l|}
$10-20$ \\
\end{tabular} & 32 & $(6,0)$ & 21 & $(6,5)$ & 11 & $(5,2)$ & \multirow{8}{*}{0,654} \\
\hline & $21-30$ & 108 & $(20,0)$ & 59 & $(18,5)$ & 49 & $(22,2)$ & \\
\hline & $31-40$ & 141 & $(26,4)$ & 81 & $(25,5)$ & 60 & $(27,8)$ & \\
\hline & $41-50$ & 140 & $(26,2)$ & 90 & $(28,4)$ & 50 & $(23,2)$ & \\
\hline & $51-60$ & 51 & $(9,6)$ & 31 & $(9,8)$ & 20 & $(9,3)$ & \\
\hline & $61-70$ & 30 & $(5,5)$ & 14 & $(4,4)$ & 16 & $(7,2)$ & \\
\hline & 71-80 & 26 & $(4,7)$ & 17 & $(5,5)$ & 8 & $(3,6)$ & \\
\hline & $81-90$ & 8 & $(1,5)$ & 5 & $(1,5)$ & 3 & $(1,5)$ & \\
\hline \multirow{4}{*}{$\begin{array}{l}\text { PRIORIDAD } \\
\text { ASIGNADA }\end{array}$} & $\begin{array}{l}\text { Emergencia, la vida del paciente } \\
\text { corre peligro o una función vital } \\
\text { si no se actúa de forma inmediata } \\
\text { y eficaz }\end{array}$ & 20 & $(3,7)$ & 11 & $(3,5)$ & 9 & $(4,1)$ & \multirow{4}{*}{0,888} \\
\hline & $\begin{array}{l}\text { Urgencia no demorable. Se debe } \\
\text { actuar lo más rápido posible pero } \\
\text { la vida no corre peligro }\end{array}$ & 496 & $(92,7)$ & 295 & $(92,8)$ & 201 & $(92,6)$ & \\
\hline & Urgencia demorable & 15 & $(2,8)$ & 10 & $(3,1)$ & 5 & $(2,3)$ & \\
\hline & \begin{tabular}{|l|} 
No urgente \\
\end{tabular} & 4 & $(0,7)$ & 2 & $(6,0)$ & 2 & $(0,9)$ & \\
\hline \multirow{12}{*}{ MES } & Enero & 54 & $(10,1)$ & 37 & $(11,6)$ & 17 & $(7,8)$ & \multirow{12}{*}{0,028} \\
\hline & Febrero & 46 & $(8,6)$ & 33 & $(10,4)$ & 13 & $(6,0)$ & \\
\hline & Marzo & 58 & $(10,8)$ & 35 & $(11,0)$ & 23 & $(10,6)$ & \\
\hline & Abril & 52 & $(9,7)$ & 35 & $(11,0)$ & 17 & $(7,8)$ & \\
\hline & Mayo & 52 & $(9,7)$ & 31 & $(9,7)$ & 21 & $(9,7)$ & \\
\hline & Junio & 39 & $(7,3)$ & 23 & $(7,2)$ & 16 & $(7,4)$ & \\
\hline & Julio & 42 & $(7,9)$ & 18 & $(5,7)$ & 24 & $(11,1)$ & \\
\hline & Agosto & 27 & $(5,0)$ & 19 & $(6,0)$ & 8 & $(3,7)$ & \\
\hline & Septiembre & 39 & $(7,3)$ & 18 & $(5,7)$ & 21 & $(9,7)$ & \\
\hline & \begin{tabular}{|l|} 
Octubre \\
\end{tabular} & 38 & $(7,1)$ & 15 & $(4,7)$ & 23 & $(10,6)$ & \\
\hline & Noviembre & 50 & $(9,3)$ & 31 & $(9,7)$ & 19 & $(8,8)$ & \\
\hline & Diciembre & 38 & $(7,1)$ & 23 & $(7,2)$ & 15 & $(6,9)$ & \\
\hline \multirow{7}{*}{ DÍA } & Lunes & 68 & $(12,7)$ & 42 & $(13,2)$ & 26 & $(12,0)$ & \multirow{7}{*}{0,584} \\
\hline & Martes & 67 & $(12,5)$ & 39 & $(12,3)$ & 28 & $(12,9)$ & \\
\hline & Miércoles & 81 & $(15,1)$ & 48 & $(15,1)$ & 33 & $(15,2)$ & \\
\hline & Jueves & 62 & $(11,6)$ & 30 & $(9,4)$ & 32 & $(14,7)$ & \\
\hline & Viernes & 104 & $(19,4)$ & 65 & $(20,4)$ & 39 & $(18,0)$ & \\
\hline & Sábado & 74 & $(13,8)$ & 48 & $(15,1)$ & 26 & $(12,0)$ & \\
\hline & Domingo & 79 & $(14,8)$ & 46 & $(14,5)$ & 33 & $(15,2)$ & \\
\hline \multirow{3}{*}{$\begin{array}{l}\text { RANGO } \\
\text { HORARIO }\end{array}$} & 08:00-15:59 & 188 & $(35,1)$ & 109 & $(34,3)$ & 79 & $(36,4)$ & \multirow{3}{*}{0,205} \\
\hline & 16:00-23:59 & 233 & $(43,6)$ & 135 & $(41,8)$ & 100 & $(46,1)$ & \\
\hline & 24:00-07:59 & 114 & $(21,3)$ & 76 & $(23,9)$ & 38 & $(17,5)$ & \\
\hline \multirow{4}{*}{$\begin{array}{l}\text { DISTRITO } \\
\text { SANITARIO }\end{array}$} & Granada Sur & 89 & $(16,6)$ & 55 & $(17,3)$ & 34 & $(15,7)$ & \multirow{4}{*}{0,142} \\
\hline & Área Metropolitana & 199 & $(37,2)$ & 129 & $(40,6)$ & 70 & $(32,3)$ & \\
\hline & Granada Nordeste & 30 & $(5,6)$ & 17 & $(5,3)$ & 13 & $(6,0)$ & \\
\hline & Granada & 217 & $(40,6)$ & 117 & $(36,8)$ & 100 & $(46,1)$ & \\
\hline
\end{tabular}


El estudio fue aprobado por el Comité Ético de Investigación Clínica del Hospital Universitario Virgen de las Nieves. Se han omitido datos privados como direcciones y teléfonos, optándose por utilizar criterios amplios como el distrito sanitario para referirnos a las zonas geográficas.

\section{RESULTADOS}

Se analizaron en total para el 2008 y 2009 respectivamente, 318 y 217 llamadas clasificadas como X84 por la operadora que atendía la demanda del Centro coordinador de Urgencias y Emergencias del Servicio Provincial 061 de Granada.

El total de llamadas telefónicas realizadas a la Central de Coordinación de Urgencias y Emergencias del Servicio Provincial 061 de los dos años estudiados, tipificadas como X84, esto es, clasificadas según el CIE $10^{13}$ con el código X84 que significa "lesión autoinflingida intencionalmente por medios no especificados", han sido 535 . De ellas, el $52,3 \%$ de intentos de suicidio lo realizaron mujeres y el 47,7 \% los hombres. Con una edad media de 41,26 $\pm 15,10$ (rango 10-90) (Tabla 1).

El 92,7 \% de los casos de nuestra muestra son urgencias no demorables, en la que se debe actuar lo más rápido posible, pero la vida no corre peligro.

De las demandas clasificadas por la operadora con el código X84 cuando aparece en el motivo literal de la demanda del usuario que telefonea el término "suicidio", se han analizado aquellas a las que acude el equipo sanitario y son de nuevo valoradas y codificadas en el lugar de los hechos.

De todas las llamadas que han sido codificadas como lesión autoinflingida intencionalmente (X84), el 19,43\% son de nuevo codificadas y de esta muestra se ratifican como intento de suicidio el 7,69\%.

De estas demandas atendidas por el equipo sanitario (esta segunda codificación se rige por el CIE $9^{14}$ ) dos asistencias (una cada año) fueron tipificadas como "muerte instantánea" (código798.1) y "muerte sin asistencia" (código798.9).

Se han encontrado diferencias estadísticamente significativas al comparar los datos de los dos años respecto del mes elegido $(p=0,28)$ (Tabla 1$)$.

En el año 2008, el 49,37\% de personas que hicieron un intento de suicidio fueron derivadas al servicio de Urgencias del hospital de referencia para su ingreso, en el 2009 el porcentaje aumenta hasta el $52,07 \%$, a pesar de que el número de intentos es menor que en el año anterior. La prioridad asignada sin embargo no se ve modificada.

No se han encontrado diferencias estadísticamente significativas por el sexo de los sujetos en ningún caso, incluso respecto de la prioridad asignada por lo que la gravedad del intento es la misma (Tabla 2). 
Tabla 2. Descripción de la muestra según sexo

\begin{tabular}{|c|c|c|c|c|c|c|}
\hline \multirow{3}{*}{ AÑO } & \multirow{3}{*}{\begin{tabular}{|l|}
2008 \\
2009
\end{tabular}} & \multicolumn{2}{|c|}{$\begin{array}{c}\text { MUJER } \\
\text { n (\%) }\end{array}$} & \multicolumn{2}{|c|}{$\begin{array}{c}\text { HOMBRE } \\
\text { n (\%) }\end{array}$} & \multirow{3}{*}{$\begin{array}{c}\begin{array}{c}\boldsymbol{p} \\
\text { mujer/hombre }\end{array} \\
0,395\end{array}$} \\
\hline & & 143 & $(51,6)$ & 134 & $(48,4)$ & \\
\hline & & 105 & $(53,3)$ & 92 & $(46,7)$ & \\
\hline \multirow{8}{*}{ EDAD (años) } & $10-20$ & 12 & $(4,9)$ & 16 & $(7,2)$ & \multirow{8}{*}{0,146} \\
\hline & 21-30 & 44 & (18) & 49 & $(21,7)$ & \\
\hline & $31-40$ & 67 & $(27,5)$ & 56 & $(24,8)$ & \\
\hline & $41-50$ & 62 & $(25,4)$ & 60 & $(26,5)$ & \\
\hline & 51-60 & 25 & $(10,2)$ & 20 & $(8,8)$ & \\
\hline & $61-70$ & 19 & $(7,8)$ & 7 & $(3,1)$ & \\
\hline & $71-80$ & 9 & $(3,7)$ & 12 & $(5,3)$ & \\
\hline & $81-90$ & 6 & $(2,5)$ & 1 & $(0,4)$ & \\
\hline \multirow{4}{*}{$\begin{array}{l}\text { PRIORIDAD } \\
\text { ASIGNADA }\end{array}$} & $\begin{array}{l}\text { Emergencia, la vida del paciente corre } \\
\text { peligro o una función vital si no se actúa } \\
\text { de forma inmediata y eficaz }\end{array}$ & 5 & (2) & 13 & $(5,8)$ & \multirow{4}{*}{0,179} \\
\hline & $\begin{array}{l}\text { Urgencia no demorable. Se debe actuar lo } \\
\text { más rápido posible pero la vida no corre } \\
\text { peligro }\end{array}$ & 233 & (94) & 206 & $(91,2)$ & \\
\hline & \begin{tabular}{|l|} 
Urgencia demorable \\
\end{tabular} & 8 & $(3,2)$ & 5 & $(2,2)$ & \\
\hline & No urgente & 2 & $(0,8)$ & 2 & $(0,9)$ & \\
\hline \multirow{12}{*}{ MES } & Enero & 31 & $(12,5)$ & 17 & $(7,5)$ & \multirow{12}{*}{0,391} \\
\hline & Febrero & 18 & $(7,3)$ & 22 & $(9,7)$ & \\
\hline & Marzo & 21 & $(8,5)$ & 33 & $(4,6)$ & \\
\hline & Abril & 19 & $(7,7)$ & 25 & $(11,1)$ & \\
\hline & Mayo & 25 & $(10,1)$ & 21 & $(9,3)$ & \\
\hline & Junio & 16 & $(6,5)$ & 16 & $(7,1)$ & \\
\hline & Julio & 22 & $(8,9)$ & 16 & $(7,1)$ & \\
\hline & Agosto & 14 & $(5,6)$ & 9 & $(4,0)$ & \\
\hline & Septiembre & 21 & $(8,5)$ & 14 & $(6,2)$ & \\
\hline & Octubre & 19 & $(7,7)$ & 17 & $(7,5)$ & \\
\hline & Noviembre & 23 & $(9,3)$ & 22 & $(9,7)$ & \\
\hline & Diciembre & 19 & $(7,7)$ & 14 & $(6,2)$ & \\
\hline \multirow{7}{*}{ DÍA } & Lunes & 37 & $(14,9)$ & 26 & $(11,5)$ & \multirow{7}{*}{0,359} \\
\hline & Martes & 34 & $(13,7)$ & 27 & $(11,9)$ & \\
\hline & Miércoles & 32 & $(12,9)$ & 39 & $(17,3)$ & \\
\hline & \begin{tabular}{|l|} 
Jueves \\
\end{tabular} & 22 & $(8,9)$ & 32 & $(14,2)$ & \\
\hline & Viernes & 50 & $(20,2)$ & 45 & $(19,9)$ & \\
\hline & Sábado & 35 & $(14,1)$ & 29 & $(12,8)$ & \\
\hline & Domingo & 38 & $(15,3)$ & 28 & $(12,4)$ & \\
\hline \multirow{3}{*}{$\begin{array}{l}\text { RANGO } \\
\text { HORARIO }\end{array}$} & 08:00-15:59 & 76 & $(30,6)$ & 88 & $(38,9)$ & \multirow{3}{*}{0,138} \\
\hline & 16:00-23:59 & 119 & (48) & 91 & $(40,3)$ & \\
\hline & 24:00-07:59 & 53 & $(21,4)$ & 47 & $(20,8)$ & \\
\hline \multirow{4}{*}{$\begin{array}{l}\text { DISTRITO } \\
\text { SANITARIO }\end{array}$} & Granada Sur & 42 & $(16,9)$ & 38 & $(16,8)$ & \multirow{4}{*}{0,745} \\
\hline & \begin{tabular}{|l|} 
Área Metropolitana \\
\end{tabular} & 97 & $(39,1)$ & 80 & $(35,4)$ & \\
\hline & Granada Nordeste & 12 & $(4,8)$ & 15 & $(6,6)$ & \\
\hline & Granada & 97 & $(39,1)$ & 93 & $(41,2)$ & \\
\hline
\end{tabular}


Existe significación estadística en la relación entre rango de edad y franja horaria de los intentos de suicidio $(p=0,018)$. A partir de los 71 años la franja horaria ele- gida para realizar una conducta suicida es desde las 8 de la mañana hasta las 15:59. Para todos los rangos de edad anteriores es desde las 16:00 h. hasta las 23:59 (Fig. 1).

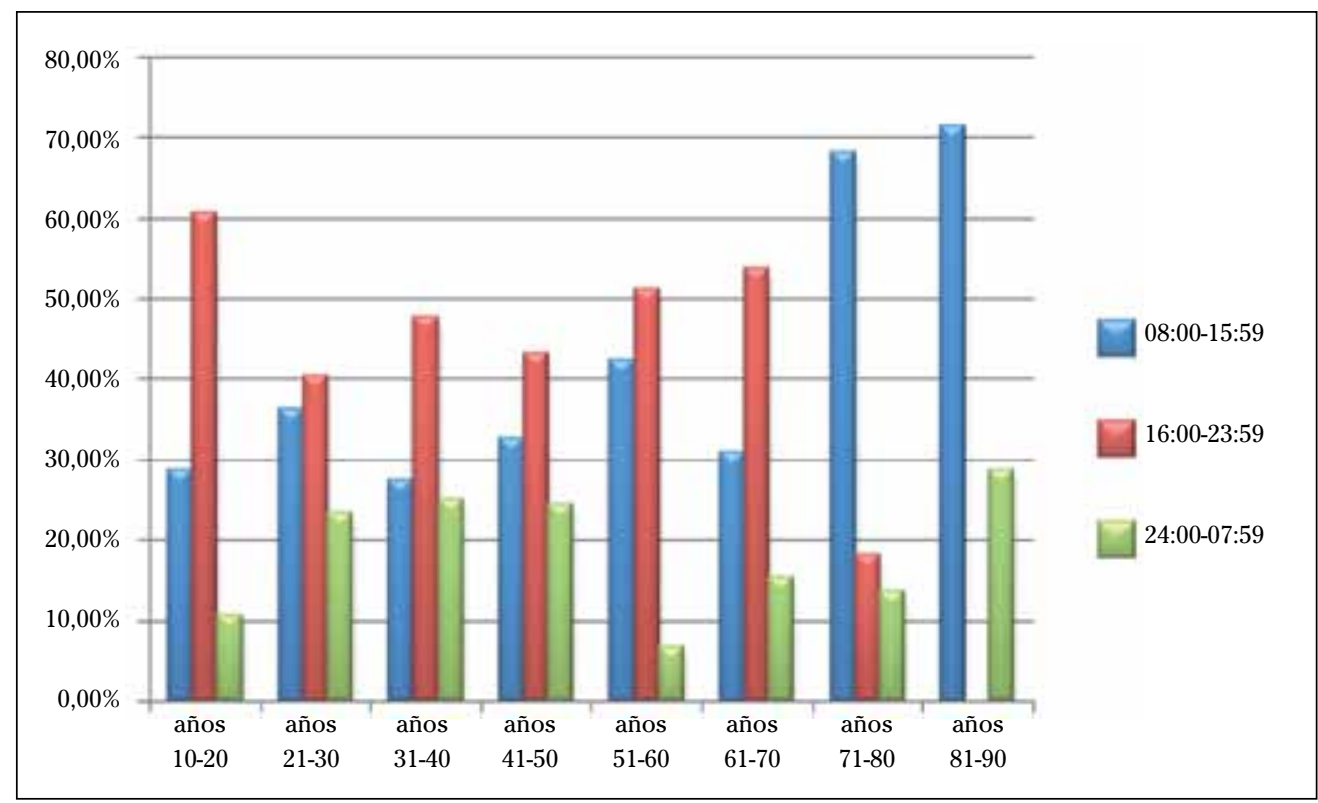

Figura 1. Intentos de suicidio según rango horario y franja de edad.

El número de personas que han realizado más de un intento en nuestra muestra han sido 11 (6 mujeres y 5 hombres) en el año 2008 y 3 personas ( 1 mujer y 2 hombres) en el año 2009. El total para los dos años es $2,61 \%$.

Tabla 3. Reintentos de suicidio

\begin{tabular}{|l|c|c|}
\hline \multicolumn{2}{|c|}{ Muestra total } & Reintentos: n(\%) \\
\hline \multirow{3}{*}{ Año } & $2008: 318$ & $11(3,45)$ \\
\cline { 2 - 3 } & $2009: 217$ & $3(1,38)$ \\
\hline Sexo & Mujer: 248 & $7(2,82)$ \\
\cline { 2 - 3 } & Hombre: 126 & $7(3,09)$ \\
\hline \multirow{5}{*}{ Edad } & $10-20: 28$ & $2(7,14)$ \\
\cline { 2 - 3 } & $21-30: 93$ & $2(1,07)$ \\
\cline { 2 - 3 } & $31-40: 123$ & $1(1,23)$ \\
\cline { 2 - 3 } & $41-50: 122$ & $7(5,83)$ \\
\cline { 2 - 3 } & $51-60: 45$ & $1(0,45)$ \\
\cline { 2 - 3 } & $61-70: 26$ & 0 \\
\cline { 2 - 3 } & $71-80: 21$ & $1(0,21)$ \\
\cline { 2 - 3 } & $81-90: 7$ & 0 \\
\hline
\end{tabular}




\section{DISCUSIÓN}

El número de intentos de suicidio con final no mortal de la provincia de Granada que hemos analizado, es aproximativo. Hemos encontrado que existe una dificultad para diagnosticar esta conducta por el personal sanitario de urgencias y emergencias. El 19,43\% de todas las llamadas que han sido codificadas como lesión autoinflingida intencional (X84) son de nuevo codificadas por el médico que las atiende in situ, confirmándose sólo el 7,69\% como acto suicida. El comportamiento suicida es complicado de determinar para estos profesionales, especialmente es difícil averiguar la voluntariedad o la intención del intento de suicidio cuando el paciente no lo expresa, cuando no puede hacerlo y cuando no queda claro por las circunstancias que rodean el acto.

En este trabajo se han descartado aquellas llamadas en las que no aparecía el término suicidio, con la certeza de perder los casos que se han diagnosticado como traumatismo (al precipitarse de un nivel a otro), accidente de tráfico, envenenamiento y abuso por medicación, que han sido ocasionados voluntariamente por el sujeto, pero de los que no se ha comunicado esta intencionalidad, ni en el motivo de la asistencia, ni en el diagnóstico in situ del profesional.

Además, no se han explorado los intentos de suicidio no mortal que han acudido directamente a algún servicio de urgencias hospitalario o del centro de salud más cercano, ni aquellos que no se han puesto en contacto con ningún servicio de urgencias por no requerir atención médica (solo el $25 \%$ de los intentos de suicidio contactan con un hospital público) ${ }^{3}$. Bodega y col $^{15}$ aseguran que una tercera parte de los que intentan suicidarse posteriormente fueron tratados en un centro de salud.

Pajonky col $^{16}$ resaltan la importancia de diagnosticar y reconocer en el paciente el intento de suicidio e ideación suicida por el personal de urgencias para prevenir la recaídas del comportamiento suicida. Su estudio revela que el diagnóstico y la documentación de los intentos de suicidio e ideación suicida siguen siendo pobres.
Según nuestros datos, en el año 2008 hubo 103 muertes por suicidio en la provincia de Granada y fueron atendidas 318 llamadas en la Central de Coordinación de Urgencias y Emergencias del Servicio Provincial 061 , por "lesión autoinflingida intencionalmente por medios no especificados"; en el año 2009, 109 muertes por suicidio y 217 llamadas o demandas telefónicas, esto es, se sucedieron dos intentos por cada suicidio. Las tasas de suicidio o de intentos de suicidio son diferentes entre los distintos países ${ }^{17}$. Entre los países que informan acerca del suicidio a la Organización Mundial de Salud las tasas más altas están en Europa Oriental. Entre los países que han experimentado un aumento significativo durante el siglo XX está España. La tasa mundial de mortalidad anual se aproxima al 14,5 por 100.000 habitantes. Hay estudios que evidencian que del $30 \%$ al $60 \%$ de las muertes suicidas fueron precedidas por intentos $^{18,19}$.

Teniendo en cuenta estas proporciones, y considerando los resultados de Niméus y $\mathrm{col}^{20}$ en los que se comprueba que el riesgo de suicidio es más evidente durante el primer año, es necesario intervenir mediante la prevención de las recaídas o los reintentos.

En el estudio europeo sobre el suicidio de Schmidtke y coll $^{21}$, las mujeres presentan mayores tasas de intentos de suicidio en una proporción de 1,5/1. En España el riesgo en la ideación y elaboración de un plan suicida es mayor en las mujeres, aunque el riesgo de realizar un intento de suicidio es similar al del hombre según los datos del estudio de Gabilondo y $\mathrm{col}^{4}$. Nuestro estudio coincide con el de Gabilondo y col y otros estudios españoles en los que el intento de suicidio es mayor en la mujer aunque sin significación estadística ${ }^{22,23}$.

El estudio de Nock $^{24}$, realizado en 17 países, concluye que entre los factores de riesgo del comportamiento suicida se encuentra el "ser del sexo femenino" y "ser joven (entre 18 y 49 años)", características concordantes con nuestros resultados.

Los factores ambientales pueden tener una influencia en el comportamiento humano y también la estacionalidad ha estado 
vinculada a las descompensaciones de los cuadros afectivos ${ }^{5,25,26}$. En el estudio llevado a cabo en Estados Unidos por Kposowa y D'Auria ${ }^{27}$ sobre suicidios consumados, el jueves y viernes son los días con menos suicidios y el verano la estación con más probabilidad. Nuestros datos no coinciden ni en el día (viernes para ambos sexos) ni en los meses (marzo y abril para los hombres, enero para las mujeres, mínimo en agosto para ambos sexos). En este sentido hay que destacar que la mayoría de los estudios consultados se refieren a datos sobre conductas suicidas mortales, mientras que los nuestros son de conductas suicidas no mortales.

Con respecto al intervalo horario en el que se producen más suicidios, en el estudio de Romero Palanco y col $^{25}$ la mayoría de los suicidios se producen entre las 8 y 16 horas, aunque en este caso no lo relaciona con la edad; en nuestro estudio, esta franja es la más frecuente en las personas de más de 70 años. Nuestros resultados coinciden con dicho estudio en que el horario donde se producen menos intentos de suicidio es el comprendido entre la 1:00 y las 7:00 AM.

Por otra parte, en nuestro estudio, la significación estadística que se produce al comparar los datos por meses de ambos años, puede explicarse porque no hay un comportamiento homogéneo, de manera que en los mismos meses cada año se han producido cifras diferentes. Sería necesario estudiar esta variable en periodos de tiempo mucho más amplios para poder observar su comportamiento.

En este sentido, nuestros resultados en ambos años indican que en el mes de agosto (verano) se contabilizan cifras más bajas de intentos de suicidio. En España, este mes se caracteriza por tener días largos, con muchas horas de exposición a la luz solar, y en el que las relaciones sociales y familiares se intensifican notablemente. Todo ello contrario al patrón observado por otros autores en cuanto a estacionalidad. Es posible que estas diferencias se expliquen por la influencia de características culturales específicas de un lugar o una comunidad, por lo que sería necesario profundizar en el estudio de la relación en- tre las conductas suicidas y los contextos culturales.

Algunos estudios ${ }^{9,28,29}$ demuestran que más de la mitad de los pacientes que realizan una conducta suicida lo habían intentado con anterioridad. En nuestra muestra el porcentaje es bastante menor. Este resultado puede deberse al sistema elegido para identificar los reintentos, que en nuestro caso ha sido manual (mediante búsqueda de coincidencias del domicilio, sexo y edad, en la base de datos de dos años), pues no se reconocen en la base de datos SIEPES. Pero también puede explicarse si tenemos en cuenta que en nuestra muestra, la gravedad de los intentos es en un 48,93\% de los casos de carácter leve, esto es, no han necesitado derivación a los servicios de urgencias de los hospitales de referencia, de manera que pueden ser conductas con un plan de suicidio poco elaborado. Otra posible explicación es que el intervalo entre ambos momentos del análisis es escaso.

La caracterización de los intentos de suicidio en nuestra provincia ofrece información relevante, a pesar de sus limitaciones, y ayuda a determinar alguno de los parámetros en los que habrá que sustentar un futuro programa de intervención psicosocial para la prevención de estas conductas ajustado a los perfiles específicos de nuestra población.

Queda por determinar la relación con la enfermedad mental, aspecto éste que dada su enorme complejidad supera los objetivos de este trabajo.

Este estudio es de carácter exploratorio y no pretende la generalización de sus resultados. Tampoco pretende explicar la realidad ni los hechos, sino describir con datos objetivos el estado de la cuestión en un lugar concreto y en un momento concreto.

Otra limitación se relaciona con las pérdidas de casos debido a dificultades para evaluar por teléfono el comportamiento suicida, a que no han necesitado asistencia sanitaria, pacientes que acuden directamente al centro de salud o urgencias del hospital; y a accidentes de tráfico, envenenamiento, abuso de medicación o traumatismos de los que no se conocen su intencionalidad. 


\section{Agradecimientos}

Al servicio de Urgencias y Emergencias 061 de la provincia de Granada.

\section{BIBLIOGRAFÍA}

1. OMS Prevención del suicidio. SUPRE Estudio de Intervención Multicentro sobre comportamientos suicidas-SUPRE-MISS: Protocolo de SUPRE-MISS. Organización Mundial de la Salud 2002. Documento WHO/MSD/ MBD/02.1. (citado 1 agosto 2010) Disponible en: http://www.who.int/mental_health/ prevention/suicide/en/supre_miss_protocol_spanish.pdf

2. Peden M, McGree K, Krug E (Eds.) Injury: a leading cause of the global burden of disease 2000. Geneve, World Health Organization 2002. ISBN 9241562323 (NLM 700). (Citado 17 diciembre 2010). Disponible en: http://whqlibdoc.who.int/publications/2002/9241562323.pdf

3. Bertolote J, Lester D. Capítulo VII: la Violencia autoinflingida. En: Informe mundial sobre la violencia y la salud. Publicación Científica y Técnica No. 588 Organización Panamericana de la Salud, Oficina Sanitaria Panamericana, Oficina Regional de la Organización Mundial de la Salud 525 Twenty-third St., NW Washington, D.C. 20037, E.U.A. 2003 p. 20131 (citado 14 agosto 2010) Disponible en: http://www.paho.org/Spanish/DD/PUB/Violencia_2003.htm

4. Gabilondo A, Alonso J, Pinto-Meza A, Vilagut G, Fernández A, Serrano-Blanco A et al. Prevalencia y factores de riesgo de las ideas, planes e intentos de suicidio en la población general española. Resultados del estudio ESEMeD. Med Clin (Barc) 2007; 129: 494-500.

5. Johnston AK, Pirkis JE, Burgess PM. Suicidal thoughts and behaviours among Australian adults: findings from the 2007 National Survey of Mental Health and Wellbeing. Aust N Z J Psychiatry 2009; 43: 635-643.

6. Department of Health 2002. National Suicide Prevention Strategy for England. Produced by the Department of Health № 29158. 2002 (citado 27 mayo 2010) Disponible en: http:// www.dh.gov.uk/en/Publicationsandstatistics/Publications/PublicationsPolicyAndGuidance/DH_4009474

7. Russell D, Turner RJ, Joiner TE. Physical disability and suicidal ideation: a communitybased study of risk/protective factors for suicidal thoughts. Suicide Life Threat Behav 2009; 39: 440-451.
8. Ribeiro JD, JoINER JE. The InterpersonalPsychological Theory of Suicidal Behavior: Current Status and Future Directions. Journal of Clinical Psychology, 2009; 65: 1291299 Published online in Wiley InterScience (www.interscience.wiley.com). DOI: 10.1002/ jclp.20621

9. Hawton K, van Heeringen K. Suicide. Lancet 2009; 373: 1372-1381.

10. Informe sobre la salud en el mundo 2001. Salud mental: nuevos conocimientos, nuevas esperanzas. ISBN 9243562010 (Clasificación NLM: WA 540.1). ISSN 1020-6760. Organización Mundial de la Salud 2001. (citado 22 junio 2010) Disponible en: http://www.who. int/whr/2001/es/index.html

11. Jané-Llopis E, Anderson P. (Eds). Mental health promotion and mental disorder prevention across European Member States: a collection of country stories. Luxembourg: European Communities. 2006 ISBN-10: 92-79-01160-X

12. Оhayon M. About suicide and its prevention. $\mathrm{J}$ Psychiatr Res 2009; 43: 885-886.

13. Lista de códigos CIE 10 (citado 10 septiembre 2010) Disponible en: http://es.wikipedia. org/wiki/Lista_de_c\%C3\%B3digos_CIE-10\#P. C3.A1ginas_web_oficiales_de_la_OMS_de_ la_CIE

14. Clasificación Internacional de Enfermedades. $9^{\mathrm{a}}$ Revisión. Modificación Clínica. eCIE9MC. Edición electrónica de la CIE-9-MC. $7^{\mathrm{a}}$ Edición (Enero 2010). Versión 1.1.0-19/02/2010. Ministerio de Sanidad y Política Social. Agencia de Calidad del Sistema Nacional de Salud. Instituto de Información Sanitaria. (citado 10 septiembre 2010). Disponible en: www.msps.es

15. Botega NJ, Azevedo Barros MB, Bosco de Olivelra H, Dalgalarrondo P, Marín-León L. Suicidal behavior in the community: Prevalence and factors associated with suicidal ideation. Rev Bras Psiquiatr 2005; 27: 45-53.

16. Pajonk FG, Schmitt P, Biedler A, Richter JC, MeYER W, LUIz T et al. Psychiatric emergencies in prehospital emergency medical systems: a prospective comparison of two urban settings. Gen Hosp Psych 2008; 30: 360-366.

17. García-Resa E, Braquehais D, Blasco H, Ramirez A, JimÉnez L, Díaz-SAStre C et al. Sociodemographic features of suicide attempts. Actas Esp Psiquiatri 2002; 30: 112-119.

18. Моsсіскі EK. Identification of suicide risk factors using epidemiologic studies. Psychiatr Clin North Am 1999; 20: 499-517.

19. Palacio-Acosta C, García-Valencia J, Diago-García J, Zapata C, Ortiz-Tobón J et al. Characte- 
ristics of people committing suicide in Medellín, Colombia. Rev Salud Pública (Bogotá) 2005; 7: 243-253.

20. NimÉus A, AlsÉn M, TräSKman-Bendz L. La Escala de Evaluación del Suicidio: un instrumento que evalúa el riesgo de suicidio de personas que han intentado quitarse la vida. Eur Psychiatry (Ed Esp) 2001; 8: 54-62.

21. Schmidtke A, Bille-Brahe U, Deleo D, Kerhof A, BJerke T, Crepet P et al. Attemped suicide in Europe: rates, trends and sociodemographic characteristics of suicide attempers during the period 1989-1992. Results of the WHO/ EURO Multicentre study on Parasuicide. Acta Psychiatr Scand 1996; 93: 327-338.

22. IBÁÑEZ FLETA E. Factores asociados a intentos autolíticos y clases diagnósticas de enfermería, en enfermos mentales hospitalizados. Biblioteca Lascasas, 2009; 5(1). Disponible en http://www.index-f.com/lascasas/documentos/lc0403.php

23. López P, González-Pinto A, Mosquera F, Aldama A, González C, Fernández de Corres B et al. Estudio de los factores de riesgo de la conducta suicida. Análisis de la atención sanitaria. Investigación Comisionada. Vitoria-Gasteiz. Departamento de Sanidad, Gobierno Vasco, 2007. Informe $\mathrm{n}^{\circ}$ : Osteba D-07-02.
24. Nock MK. Cross-national prevalence adn risk factors for suicidal ideation, plans and attemps. Br J Psychiatry 2008; 192, 98-105.

25. Romero Palanco JL, Gamero Lucas JJ, Martínez García P. Aspectos epidemiológicos del suicidio consumado en la provincia de Cádiz (1999-2003). Cuad Med Forense 2007; 13: 3344.

26. SAn Gil Martín J, GonzÁlez de Rivera JL, GonzÁLEz González J. Estacionalidad y Psicopatología. Psiquis Vol. IX/88: 92-101.

27. Kposowa AJ, D'Auria S. Association of temporal factors and suicides in the United States, 2000-2004. Soc Psychiatry Psychiatr Epidemiol 2010; 45: 433-445.

28. Lozano C, Huertas A, Martínez CJ, Ezquiaga E, García E, Rodríguez FJ. Estudio descriptivo del parasuicidio en las urgencias psiquiátricas. Rev Asoc Esp Neuropsiq [revista electrónica]. 2004 [citado 23 septiembre 2010]; 91: 11-22. Disponible en: http://scielo.isciii.es/ scielo.php?script $=$ sci_arttext $\&$ pid $=$ S0211 57352004000300002\&lng=es. doi: 10.4321/ S0211-57352004000300002

29. Cabrera JF, Jiménez V, Páez M. Las conductas suicidas en Urgencias. Puesta al día en urgencias, emergencias y catástrofes. Dialnet 2008; 8: 97. 